\title{
Modelling Intervalling Effect of High Frequency Trading on Portfolio Volatility
}

\author{
Ki Hoon Hong \\ College of Business Administration, Hongik University, Seoul, South Korea \\ Email: hong.jimmy@gmail.com
}

How to cite this paper: Hong, K.H. (2019) Modelling Intervalling Effect of High Frequency Trading on Portfolio Volatility. Theoretical Economics Letters, 9, 2362-2370. https://doi.org/10.4236/tel.2019.97150

Received: July 31, 2019

Accepted: September 24, 2019

Published: September 27, 2019

Copyright $\odot 2019$ by author(s) and Scientific Research Publishing Inc. This work is licensed under the Creative Commons Attribution International License (CC BY 4.0).

http://creativecommons.org/licenses/by/4.0/

\begin{abstract}
This paper theoretically examines whether reducing the minimum trading interval could affect portfolio volatility. Modelling the underlying de-trended asset price with Ornstein Uhlenbeck process, the paper investigates the volatility of portfolios that employ buy and hold strategy and momentum strategy. The paper presents theoretical evidence that the fast trading could increase portfolio price fluctuation and hence potentially suggests another cost of high frequency trading, besides the well-known damages including herding, aggressive trading strategies dark pools, immediate-or-cancel type orders.
\end{abstract}

\section{Keywords}

Volatility, Fast Trading, Momentum Trading, Time Series Momentum, Intervalling Effect

\section{Introduction}

When portfolio managers execute a trade, they typically decide two components of a trade: trading rule and trading frequency. Trading rule is the strategy implemented by portfolio managers. Momentum or contrarian strategies are good examples. Any trading rule can be implemented at any trading frequency. For example, a manager who rebalances portfolio holdings annually can employ the same trading rule when rebalancing on quarterly basis. There is no reason why the same strategy cannot be employed on intraday basis.

High frequency trading (HFT) also has two distinctive components, trading strategy and fast trading component. The impact of the two components should be distinguished, at least in theory. Technically speaking, one could execute any trading rule very fast by shortening the available trading interval. However, careful reading of the existing literature reveals that most of the criticisms are based on the trading strategies of HFT (see [1] [2] [3]). One example is that the 
algorithms, programmed similarly, create herding and weaken the stability of the financial markets. There is less emphasis on potential change in volatility due to the statistical properties of fast trading. In order to investigate potential influence of fast trading on portfolio volatility, trading rule needs to be controlled. This can be very hard with empirical analysis; therefore this paper proposes a theoretical model of trading interval, controlling the trading strategies to investigate the sensitivity of portfolio volatility.

This paper is related to two different literatures. One is intervalling effect and the other is HFT.

\subsection{Intervalling Effect Literature}

Empirically and theoretically, it is known that beta estimates are sensitive to the data measurement frequency, which is referred to intervalling effect. [4] found that beta estimates are dependent on the length of the differencing interval. [5] provided a theoretical explanation of intervalling based on iid multiplicative returns. There are many other contributions including [6] [7] and [8].

\subsection{High Frequency Trading Literature}

HFT is generally believed to lower the cost of trading, increase the informativeness of quotes, provide liquidity and eliminate arbitrage (see [9]) through fast trading. There are many claimed costs of HFT including increased volatility, herding, aggressive trading strategies dark pools, immediate-or-cancel type orders. This paper tackles the first claim but from the portfolio managers' perspective. The majority of the existing studies investigated whether HFT results in increased volatility in the market. However, another relevant question would be whether it increases portfolio price fluctuation.

This paper finds that the portfolio volatility increases as the minimum length of the trading interval decreases. This could be interpreted as an indication that the fast trading component of HFT could increase portfolio level volatility. The theoretical finding of the paper suggests there could be another potential cost of HFT, in purely statistical terms, besides the well-known damages of HFT including herding, aggressive trading strategies dark pools, immediate-or-cancel type orders and many others.

This paper contributes to the existing literature in the following aspects. First, the paper builds a theoretical model that can separate the impact of fast trading component of HFT from algorithmic trading strategy component. The second contribution is that the paper brings the discussion to portfolio level where most of existing works focus on the market level investigation. Portfolio level analysis would be more relevant to investors and portfolio managers. The paper provides a theoretical support that the availability of fast trading could contribute to increase in portfolio volatility. The third contribution is that it expands the scope of intervalling effect investigation to momentum trading strategy.

The rest of the paper is organized as follows. Section 2 provides the model 
used in this paper. Section 3 presents the impact of fast trading. Section 4 concludes the paper.

\section{The Model}

Let de-meaned $\log$ price process $q(t)=\log p(t)-\mu t$ has distribution $q(t) \sim\left(0, \sigma^{2}\right)$. Denote the autocorrelation between $q(t)$ and $q(t+1)$ as $\rho_{A}(h)$. Assume

$$
\mathrm{d} q(t)=-\theta q(t) \mathrm{d} t+\sigma \mathrm{d} W(t)
$$

where $\theta$ and $\sigma$ are the parameters of the Ornstein Uhlenbeck (OU) process. Assume $\sigma>0$. $W(t)$ denotes a Wiener process. The OU process is often used to model mean reverting financial processes. Mean reverting processes are naturally attractive to model financial asset prices because of the economic argument that when prices are "too high", demand will reduce, and supply will increase prices. The use of this process has been studied by [10]. It has been successfully used by [11] [12] and [13] in studying financial asset price process. The analytical solution for this process is

$$
q(t+h)=\mathrm{e}^{-\theta(t+h)} q(0)+\sigma \int_{0}^{t+h} \mathrm{e}^{-\theta(t-u)} \mathrm{d} W(u)
$$

where $h$ is the time interval that price is reported hence is the minimum trading interval. Then we have

$$
E(q(t+h))=\mathrm{e}^{-\theta(t+h)} q(0) \text { and } \operatorname{Var}(q(t+h))=\sigma_{q}^{2}(h)=\left(\frac{1-\mathrm{e}^{-2 \theta(t+h)}}{2 \theta}\right) \sigma^{2}
$$

Note that the implicit assumption to keep the variances positive is $\theta>0$. This paper employs and expands the definition of the Moving Average (MA) oscillator by [14]. Let 1 stands for the time period that the MA is computed for a long position and $s$ for the time period that the MA is computed for a short position. Hence $s>l$ and they are overlapping. Denote SMA as MA over $s$ and LMA as MA over 1 .

Definition 1: The $M A(1, s)$ position can be represented as

$$
\operatorname{MA}(l, s)=\max (\delta(t), 0)
$$

where

$$
\delta(t)=\left\{\begin{array}{ll}
1 & \text { if } \operatorname{LMA}(t)-\operatorname{SMA}(t)>0 \\
0 & \text { if } \operatorname{LMA}(t)-\operatorname{SMA}(t)<0
\end{array}, \operatorname{SMA}(t)=\frac{\sum_{i=1}^{s} p(t-i+1)}{s}\right.
$$

and

$$
\operatorname{LMA}(t)=\frac{\sum_{i=1}^{l} p(t-i+1)}{l}
$$

where $p(t)$ is an asset price at time $t$. The MA rule takes advantage of time series momentum. 


\section{Impact of Fast Trading on Portfolio Volatility}

In order to compare the sensitivity of variances with respect to the minimum trading interval, $h$, unit period needs to be introduced. The unit period is the time period that the continuous OU process is originally discretized. Using the unit period, $h$ could be expressed in relative term, $1 / h$. All $h$ period variances could be compared by converting into unit period variances.

Remark 1: The unit period variance of a portfolio with Buy and Hold (BH) strategy is

$$
\sigma_{B H}^{2}(h)=\frac{1}{h} \sigma_{q}^{2}(h)=\left(\frac{1-\mathrm{e}^{-2 \theta(t+h)}}{2 \theta}\right) \frac{1}{h} \sigma^{2}
$$

Proposition 1: The sensitivity of variance of a BH strategy with respect to $h$ is negative.

The proof of Proposition 1 is in Appendix A. Proposition 1 implies that shorter minimum trading interval increases the volatility of a long position on the asset, therefore statistically speaking, the availability of fast trading could increase portfolio volatility. Practically speaking, this may explain why some stocks' return volatility suddenly increases when investors start trading the stock more frequently. Also from the market's perspective, as technology allows faster trading, market could become more volatile.

The evidence from a portfolio with $\mathrm{BH}$ strategy may not be convincing enough due to the simplicity of the portfolio component. Therefore the volatility of a portfolio with one of the most popular trading strategies, Moving Average rule, is investigated. Define the critical value of a $\operatorname{MA}(s, I)$ rule is defined as

$$
C(s, l ; t)=\frac{\operatorname{LMA}(t)}{\operatorname{SMA}(t)}=\exp \left(w^{\prime} Q(t)\right)
$$

where

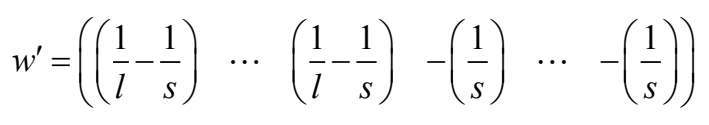

and

$$
Q(t)^{\prime}=\left(\begin{array}{llllll}
q(t) & q(t-1) & \cdots & q(t-l+1) & \cdots & q(t-s+1)
\end{array}\right)
$$

The strategy takes a long position if $C(s, l ; t)$ becomes more than 1 and liquidate the position otherwise. Assuming stationarity in the second moments

$$
C(s, l ; t)=\exp \left(w^{\prime} Q(t)\right), w^{\prime} Q(t) \sim N\left(0, w^{\prime} \Omega w\right)
$$

where $\Omega$ is the $(s \times s)$ covariance of $Q(t)$. Let $\rho_{A}(k)$ be the asset price autocorrelation between $k$ periods. Assume stationarity in second moments. Note that $\Omega$ is a vector of $q(i)$ values $(i=1, \cdots, t) . \Omega_{m}$ is a covariance matrix between $Q(t)$ and $Q(t-1)$.

Now, the OU model of de-meaned price process is applied to the technical trading rule model and investigates the variance of the trading strategy with respect to $h$. The interval that SMA is computed, $s$, varies proportionate to the size 
of $h$. For 10 day MA and when $h$ is 1 week, $s=2$, when $h$ is 1 day, $s=10$, when $h$ is 1 hour, $s=65$, when $h$ is 1 minute, $s=3900$ and when $h$ is 1 second, $s=$ 234,000. Therefore $s$ should be denoted as $s(h)$.

Proposition 2: The sensitivity of unit period variance of the trading strategy critical value, $C(t)$, with respect to $h$ is negative.

The proof of Proposition 2 is in Appendix B. Proposition 2 indicates that the volatility of a portfolio formed by the MA rule increases as fast trading becomes available. It is often argued that HFT firms increase market volatility by employing similar algorithms and create herding behaviour. This paper adds portfolio level evidence that HFT activities may increase the price volatility. Note that the result does make any indication about the effect of the automated trading system on volatility. The trading strategy is controlled to be either $\mathrm{BH}$ or MA.

\section{Conclusions}

This paper builds a simple theoretical model to investigate the intervalling effect of portfolio volatility that employs buy and hold and momentum strategies. The paper finds that the portfolio volatility increases as the minimum trading interval decreases. The finding of this paper adds portfolio level evidence that HFT activities may increase volatility. The availability of fast trading could statistically increase portfolio volatility when trading strategies are controlled. This paper contributes to the existing literature by presenting a method to distinguish the impact of trading strategy and trading frequency.

This paper theoretically investigates the intervalling effect of portfolio volatility that employs buy and hold and momentum strategies. Empirical investigation of such effect could be an interesting future research topic.

\section{Supported}

This work was supported by 2019 Hongik University Research Fund.

\section{Conflicts of Interest}

The author declares no conflicts of interest regarding the publication of this paper.

\section{References}

[1] Zhang F. (2010) The Effect of High-Frequency Trading on Stock Volatility and Price Discovery. Working Paper. https://doi.org/10.2139/ssrn.1691679

[2] Hasbrouck, J. and Saar, G. (2011) Low-Latency Trading. Working Paper. https://doi.org/10.2139/ssrn.1695460

[3] Glode, V., Green, R.C. and Lowery, R. (2012) Financial Expertise as an Arms Race. The Journal of Finance, 67, 1723-1759. https://doi.org/10.1111/j.1540-6261.2012.01771.x

[4] Pogue, G.A. and Solnik, B.H. (1974) The Market Model Applied to European Common Stocks: Some Empirical Results. Journal of Financial and Quantitative 
Analysis, 9, 917-944. https://doi.org/10.2307/2329728

[5] Levhari, D. and Levy, H. (1977) The Capital Asset Pricing Model and the Investment Horizon. The Review of Economics and Statistics, 59, 92-104.

https://doi.org/10.2307/1924908

[6] Dimson, E. (1979) Risk Measurement When Shares Are Subject to Infrequent Trading. Journal of Financial Economics, 7, 197-226. https://doi.org/10.1016/0304-405X(79)90013-8

[7] Corhay, A. (1988) The Adjustment for the Intervalling Effect Bias in Beta: A Broader and Multi Period Test. European Institute for Advanced Studies in Management, Brussels, Working Paper No. 88, 26.

[8] Handa, P., Kothari, S.P. and Wasley, C. (1993) Sensitivity of Multivariate Tests of the Capital Asset-Pricing Model to the Return Measurement Interval. Journal of Finance, 48, 1543-1551. https://doi.org/10.1111/j.1540-6261.1993.tb04767.x

[9] Hendershott, T., Jones, C.M. and Menkveld, A.J. (2010) Does Algorithmic Trading Improve Liquidity? The Journal of Finance, 66, 1-33.

https://doi.org/10.1111/j.1540-6261.2010.01624.x

[10] Bergstrom, A.R. (1990) Continuous Time Econometrics Modelling. Oxford University Press, Oxford.

[11] Lo, A.W. and Wang, J. (1995) Implementing Option Pricing Models When Asset Returns Are Predictable. Journal of Finance, 50, 87-129.

https://doi.org/10.1111/j.1540-6261.1995.tb05168.x

[12] Kramer, R. and Richter, M. (2007) A Generalized Bivariate Ornstein-Uhlenbeck Model for Financial Assets. Tagungsband zum Workshop 'Stochastische Analysis'. Chemnitz.

[13] Onalan, O. (2009) Financial Modelling with Ornstein-Uhlenbeck Processes Driven by Levy Process. Proceedings of the World Congress on Engineering, London, 1-3 July 2009, 1-6. https://doi.org/10.1007/978-90-481-8776-8_38

[14] Hong, K. and Satchell, S. (2015) Time Series Momentum Trading Strategy and Autocorrelation Amplification. Quantitative Finance, 15, 1471-1487.

https://doi.org/10.1080/14697688.2014.1000951 


\section{Appendix A: Derivation of Proposition 1}

The unit period variance of a portfolio with $\mathrm{BH}$ strategy can be rewritten as

$$
\sigma_{B H}^{2}(h)=\frac{\sigma^{2}}{2 \theta}\left(\frac{1}{h}-\mathrm{e}^{-2 \theta t} \frac{\mathrm{e}^{-2 \theta h}}{h}\right)
$$

Applying $\frac{\partial h^{-1}}{\partial h}=-h^{-2}, \frac{\partial \mathrm{e}^{-2 \theta h}}{\partial h}=\sigma^{2} \mathrm{e}^{-2 \theta(t+h)}$ and the chain and product rule, we get

$$
\begin{aligned}
\frac{\partial \sigma_{B H}^{2}(h)}{\partial h} & =\frac{\sigma^{2}}{2 \theta}\left(-h^{-2}-\mathrm{e}^{-2 \theta t}\left(\sigma^{2} \mathrm{e}^{-2 \theta(t+h)} h^{-1}-h^{-2} \mathrm{e}^{-2 \theta h}\right)\right) \\
& =\frac{\sigma^{2}}{2 \theta}\left(-\mathrm{e}^{-2 \theta t} \sigma^{2} \mathrm{e}^{-2 \theta(t+h)} h^{-1}-h^{-2}\left(1-\mathrm{e}^{-2 \theta(t+h)}\right)\right)
\end{aligned}
$$

Therefore, we have $\frac{\partial \sigma_{B H}^{2}(h)}{\partial h}<0$.

\section{Appendix B: Proof of Proposition 2}

The variance of the technical trading strategy with $m=0$ and applying $\rho_{A}(0)=1$, we get

$$
\begin{aligned}
& \sigma_{q}^{2}\left(a^{2}+b^{2}\left((s-1)+\sum_{i=1}^{s-2}(s-i-1)\left(\rho_{A}(i)+\rho_{A}(-i)\right)\right)\right. \\
& \left.+a b\left(\sum_{i=0}^{s-2} \rho_{A}(i+1)+\sum_{i=0}^{s-2} \rho_{A}(-i-1)\right)\right)
\end{aligned}
$$

Given

$$
\operatorname{Var}(q(t))=\sigma_{q}^{2}=\left(\frac{1-\mathrm{e}^{-2 \theta(t+h)}}{2 \theta}\right) \sigma^{2} \text { and } \operatorname{Corr}\left[q_{A}(t), q_{A}(t+h)\right]=\rho_{A}(h)=\mathrm{e}^{-\theta h} \text {, }
$$

we get

$$
\begin{aligned}
& \sigma^{2}\left(\frac{1-\mathrm{e}^{-2 \theta(t+h)}}{2 \theta}\right)\left(a^{2}+b^{2}\left((s-1)+\sum_{i=1}^{s-2}(s-i-1)\left(\mathrm{e}^{-\theta i}+\mathrm{e}^{\theta i}\right)\right)\right. \\
& \left.+a b\left(\sum_{i=0}^{s-2} \mathrm{e}^{-\theta(i+1)}+\sum_{i=0}^{s-2} \mathrm{e}^{\theta(i+1)}\right)\right)
\end{aligned}
$$

Denoting that $a, b$ and $s$ are dependent on $h$, we have

$$
\begin{aligned}
& \sigma^{2}\left(\frac{1-\mathrm{e}^{-2 \theta(t+h)}}{2 \theta}\right)\left(a(h)^{2}+b(h)^{2}\left((s(h)-1)+\sum_{i=1}^{s(h)-2}(s(h)-i-1)\left(\mathrm{e}^{-\theta i}+\mathrm{e}^{\theta i}\right)\right)\right. \\
& \left.+a(h) b(h)\left(\sum_{i=0}^{s(h)-2} \mathrm{e}^{-\theta(i+1)}+\sum_{i=0}^{s(h)-2} \mathrm{e}^{\theta(i+1)}\right)\right)
\end{aligned}
$$

This is $h$ period volatility. The unit period volatility can be written as

$$
\begin{aligned}
\sigma_{T}^{2}(h)= & \frac{1}{h} \sigma^{2}\left(\frac{1-\mathrm{e}^{-2 \theta(t+h)}}{2 \theta}\right)\left(a(h)^{2}+b(h)^{2}\left((s(h)-1)+\sum_{i=1}^{s(h)-2}(s(h)-i-1)\left(\mathrm{e}^{-\theta i}+\mathrm{e}^{\theta i}\right)\right)\right. \\
& \left.+a(h) b(h)\left(\sum_{i=0}^{s(h)-2} \mathrm{e}^{-\theta(i+1)}+\sum_{i=0}^{s(h)-2} \mathrm{e}^{\theta(i+1)}\right)\right)
\end{aligned}
$$




$$
\sigma_{A}^{2}(h)=\sigma^{2}\left(\frac{1-\mathrm{e}^{-2 \theta(t+h)}}{2 \theta}\right)>0 \text { and } \frac{\partial \sigma_{A}^{2}(h)}{\partial h}<0
$$

Let

$$
\begin{aligned}
\gamma(h)= & a(h)^{2}+b(h)^{2}\left((s(h)-1)+\sum_{i=1}^{s(h)-2}(s(h)-i-1)\left(\mathrm{e}^{-\theta i}+\mathrm{e}^{\theta i}\right)\right) \\
& +a(h) b(h)\left(\sum_{i=0}^{s(h)-2} \mathrm{e}^{-\theta(i+1)}+\sum_{i=0}^{s(h)-2} \mathrm{e}^{\theta(i+1)}\right)
\end{aligned}
$$

We know that $\gamma(h)>0$ and we assumed that $s(h)>2$. More specifically,

$$
\begin{aligned}
a(h)>0, \quad b(h) & <0, \quad \sum_{i=1}^{s(h)-2}(s(h)-i-1)\left(\mathrm{e}^{-\theta i}+\mathrm{e}^{\theta i}\right)>0, \quad a(h) b(h)<0, \\
\sum_{i=0}^{s(h)-2} \mathrm{e}^{-\theta(i+1)}>0, \quad \sum_{i=0}^{s(h)-2} \mathrm{e}^{\theta(i+1)}>0 & \\
\frac{\partial \gamma(h)}{\partial h}= & {\left[2 a(h) \frac{\partial a(h)}{\partial h}+2 b(h) \frac{\partial b(h)}{\partial h}(s(h)-1)+b(h)^{2} \frac{\partial s(h)}{\partial h}\right.} \\
& +2 b(h) \frac{\partial s(h)}{\partial h}\left(\sum_{i=0}^{s(h)-2} \mathrm{e}^{-\theta(i+1)}+\sum_{i=0}^{s(h)-2} \mathrm{e}^{\theta(i+1)}\right) \\
& +b(h)^{2} \sum_{i=1}^{s(h)-2} \frac{\partial s(h)}{\partial h}\left(\mathrm{e}^{-\theta i}+\mathrm{e}^{\theta i}\right) \\
& +\frac{\partial a(h)}{\partial h} b(h)\left(\sum_{i=0}^{s(h)-2} \mathrm{e}^{-\theta(i+1)}+\sum_{i=0}^{s(h)-2} \mathrm{e}^{\theta(i+1)}\right) \\
& \left.+\frac{\partial b(h)}{\partial h} a(h)\left(\sum_{i=0}^{s(h)-2} \mathrm{e}^{-\theta(i+1)}+\sum_{i=0}^{s(h)-2} \mathrm{e}^{\theta(i+1)}\right)\right]
\end{aligned}
$$

Since $\frac{\partial s(h)}{\partial h}>0, \frac{\partial a(h)}{\partial h}>0, \frac{\partial b(h)}{\partial h}>0$ and $\frac{\partial a(h)}{\partial h}=\frac{\partial b(h)}{\partial h}=s(h) \frac{\partial s(h)}{\partial h}$

$$
\begin{aligned}
& \frac{\partial \gamma(h)}{\partial h}=\left[2 a(h) \frac{\partial a(h)}{\partial h}+2 b(h) \frac{\partial b(h)}{\partial h}(s(h)-1)\right. \\
& +b(h)^{2} \frac{\partial s(h)}{\partial h}+b(h)^{2} \sum_{i=1}^{s(h)-2} \frac{\partial s(h)}{\partial h}\left(\mathrm{e}^{-\theta i}+\mathrm{e}^{\theta i}\right) \\
& \left.+\frac{\partial b(h)}{\partial h} a(h)\left(\sum_{i=0}^{s(h)-2} \mathrm{e}^{-\theta(i+1)}+\sum_{i=0}^{s(h)-2} \mathrm{e}^{\theta(i+1)}\right)\right] \\
& +\left[2 b(h) \frac{\partial s(h)}{\partial h}\left(\sum_{i=0}^{s(h)-2} \mathrm{e}^{-\theta(i+1)}+\sum_{i=0}^{s(h)-2} \mathrm{e}^{\theta(i+1)}\right)\right. \\
& \left.+\frac{\partial a(h)}{\partial h} b(h)\left(\sum_{i=0}^{s(h)-2} \mathrm{e}^{-\theta(i+1)}+\sum_{i=0}^{s(h)-2} \mathrm{e}^{\theta(i+1)}\right)\right] \\
& \frac{\partial b(h)}{\partial h} a(h)\left(\sum_{i=0}^{s(h)-2} \mathrm{e}^{-\theta(i+1)}+\sum_{i=0}^{s(h)-2} \mathrm{e}^{\theta(i+1)}\right) \\
& +2 b(h) \frac{\partial s(h)}{\partial h}\left(\sum_{i=0}^{s(h)-2} \mathrm{e}^{-\theta(i+1)}+\sum_{i=0}^{s(h)-2} \mathrm{e}^{\theta(i+1)}\right)
\end{aligned}
$$




$$
\begin{aligned}
& +\frac{\partial a(h)}{\partial h} b(h)\left(\sum_{i=0}^{s(h)-2} \mathrm{e}^{-\theta(i+1)}+\sum_{i=0}^{s(h)-2} \mathrm{e}^{\theta(i+1)}\right) \\
& =\frac{\partial s(h)}{\partial h}\left(\sum_{i=0}^{s(h)-2} \mathrm{e}^{-\theta(i+1)}+\sum_{i=0}^{s(h)-2} \mathrm{e}^{\theta(i+1)}\right)(s(h) a(h)+3 b(h))>0
\end{aligned}
$$

Therefore we have $\frac{\partial \gamma(h)}{\partial h}>0$. Since $\sigma_{T}^{2}(h)=\sigma_{A}^{2}(h) \gamma(h)$, we have

$$
\frac{\partial \sigma_{T}^{2}(h)}{\partial h}=\frac{\partial \sigma_{A}^{2}(h)}{\partial h} \gamma(h)+\sigma_{A}^{2}(h) \frac{\partial \gamma(h)}{\partial h}<0
$$

